Escuela de Ciencias Sociales y Humanidades, UNED, C.R.

URL: http://investiga.uned.ac.cr/revistas/index.php/espiga/index

ISSN: 1409-4002 • e-ISSN: 2215-454X

http://dx.doi.org/10.22458/re.v17i34.1168

\title{
Nuevas relaciones de poder/saber en la psicología de las organizaciones: una aproximación desde Foucault*
}

\author{
Néstor Raúl Porras-Velásquez** \\ iD https://orcid.org/0000-0002-7488-5249
}

Recibido: 23 de diciembre, 2015 - Aceptado: 29 de marzo, 2017

\begin{abstract}
RESUMEN
El objetivo de este artículo es hacer una revisión crítica sobre los aportes de la propuesta de Michel Foucault, para el análisis de las relaciones de poder en el campo de la psicología de las organizaciones. El método utilizado fue el análisis documental de obras que abordan las conceptualizaciones sobre el poder y las bases en las que se sustenta, los modos de ejercerlo y algunas de las aplicaciones e implicaciones de dichos modelos en la gestión de los recursos humanos. Los resultados muestran las prácticas de gestión humana como el dispositivo de control y articulador de los modos de subjetivación de los individuos y de las acciones que estos ejercen sobre sí mismos, a través del empleo de las tecnologías del yo y las prácticas de gobierno sobre el cuerpo y la mente. Las conclusiones evidencian el papel fundamental del dispositivo disciplinario fabril en la construcción y constitución de unas subjetividades laborales «dóciles».
\end{abstract}

Palabras clave: psicología organizacional, relaciones de poder, tecnologías del yo, dispositivos disciplinarios de control y subjetividades laborales.

\section{Formato de citación según APA}

Porras-Velásquez, N. (2017). Nuevas relaciones de poder/saber en la psicología de las organizaciones: una aproximación desde Foucault. Revista Espiga, 16(34), 270-285. Doi: http://dx.doi.org/10.22458/re.v17i34.1168

Formato de citación según Chicago-Deusto

Porras-Velásquez Néstor-Raúl. «Nuevas relaciones de poder/saber en la psicología de las organizaciones: una aproximación desde Foucault». Revista Espiga 16, n. ${ }^{\circ} 34$ (juliodiciembre, 2017): 270-285. Doi: http://dx.doi.org/10.22458/re.v17i34.1168

* Artículo de reflexión derivado del proyecto de investigación: «Propuesta de intervención en los problemas de lo humano y su lazo social desde los hallazgos de la línea de investigación psicosis y psicoanálisis».

** Psicólogo de la Universidad Nacional de Colombia. Actualmente docente-investigador de la Fundación Universitaria los Libertadores. Universidad Nacional de Colombia. Colombia. Correo: nrporrasv@liberatores.edu.co/n.porras.69@hotmail.com 


\section{Introducción}

De acuerdo con el filósofo y psicólogo francés Michel Foucault, «no es posible que el poder se ejerza sin el saber, es imposible que el saber no engendre poder» ${ }^{1}$. En este sentido, cualquier análisis de la relaciones del poder en el campo de la psicología organizacional implica necesariamente tropezarse con una verdad que es producida siempre en contextos socioculturales concretos y con efectos sobre las subjetividades laborales. En este sentido, el presente artículo es derivado del proyecto de investigación: «Propuesta de intervención en los problemas de lo humano y su lazo social». En el que se abordan, relacionan y analizan los conceptos de poder, sujeto, discurso y dispositivo desde la perspectiva de Michel Foucault los cuales permitan comprender el lazo social que se configura en las relaciones laborales del mundo del trabajo con la intención de ampliarlos, complementarlos y replantearlos en escenarios más allá de la clínica psicoanalítica. De igual forma, con esta investigación se busca también dar continuidad a la reflexión iniciada por el autor ${ }^{2}$ sobre los discursos y prácticas de inserción laboral, la producción y la salud mental, de la misma línea de investigación, así como la responsabilidad social empresarial $^{3}$ y los efectos de estas prácticas en la subjetividad laboral ${ }^{4}$. Además, se pretende aportar elementos básicos de análisis para la comprensión de los modos de subjetivación laboral producto de relaciones de poder en la dinámica interna de las organizaciones empresariales y el lugar de la psicología como discurso que produce una verdad y un sujeto, tal como lo expresa la cita al inicio del texto.

Las relaciones de poder juegan un papel fundamental y siempre están presentes en las relaciones interpersonales que surgen en el mundo del trabajo y de las organizaciones $^{5}$. El concepto de poder ocupa un lugar muy destacado en la literatura científica de las teorías de la organización, ya que es considerado uno de los aspectos prioritarios en el estudio de la conducta y la gestión organizacional ${ }^{6}$.

Desde ahora, se puede afirmar que las organizaciones no son sistemas totalmente racionales. La mayoría de las actividades, acciones y decisiones que se toman y realizan en dichos contextos están fuertemente influenciadas por factores emocionales, subjetivos y coyunturales que pretenden adquirir, mantener, incrementar y usar el poder y otros recursos, para obtener los resultados preestablecidos por un grupo particular de personas con intereses particulares. Por esta razón, es importante señalar, desde ahora, que el poder es un fenómeno connatural e inherente a la dinámica de cualquier organización

1. Michel Foucault, Vigilar y castigar: nacimiento de la prisión (Madrid: Siglo XXI, 2005), 37.

2. Néstor Porras Velásquez, «Inserción laboral y salud mental: una reflexión desde la psicología del trabajo». Tesis psicológica 8, . $^{\circ} 2$ (2013): 98-117.

3. Néstor Porras Velásquez, «La responsabilidad social empresarial e inserción laboral del enfermo mental». En Sonia Cogollo. Responsabilidad social. Perspectivas para la acción en Colombia, 88-105, Medellín: FUNLAM, 2015.

4. Néstor Porras Velásquez, «La Psicología del trabajo en relación con la subjetividad, la inserción laboral y la salud mental». En J. Báez y Cols. Salud mental y el sistema de producción, 61-108. Bogotá: Fundación universitaria los Libertadores, 2016.

5. Jefrey Pfeffer, Managing with power (Boston Harvard Business School Press, 1992).

6. Henry Mintzberg, Power in and around organizations (Englewood Clifts, NJ: Prentice-Hall, 1993). 
y que las organizaciones son sistemas sociales constituidos por una amplia variedad de intereses, no siempre confluyentes, que por medio de un proceso de negociación pretenden articular las diferencias para lograr la meta propuesta para la organización como colectivo humano ${ }^{7}$.

Por otra parte, para cualquier profesional de la psicología que desee comprender cómo funcionan las organizaciones empresariales, cómo se establecen, mantienen y transforman las relaciones laborales y sobre todo, por qué sus miembros, individual y colectivamente, actúan como lo hacen. Así, es necesario y urgente emprender un decidido estudio del fenómeno del poder en el contexto del trabajo asalariado. De igual manera, es importante reconocer desde el comienzo que las relaciones de poder en el mundo del trabajo siempre han estado articuladas a la estructura formal e informal de las organizaciones y a los sistemas de gestión de las mismas. En consecuencia, los roles desempeñados por sus integrantes, resultan también afectados por los cambios que se van produciendo en el entorno organizacional.

El contexto, el análisis de la dinámica del poder y los procesos de influencia, así como las coaliciones que se van configurando en defensa de los intereses de grupo particular, poseen una gran incidencia en los procesos de cambio organizacional y resistencia al mismo. Estas fuerzas pueden actuar dificultando o estimulando el proceso de cambio, con el objetivo de mantener o aumentar su capacidad de poder y estatus dentro de la organización, generan profundos cambios en las relaciones laborales.

Históricamente las relaciones laborales se expresan en relaciones salariales y formas particulares de organizar el trabajo, las tareas, y distribuir jerárquicamente la autoridad y el poder entre sus integrantes, de acuerdo con el puesto que ocupan en la estructura organizacional. De esta manera, se fundan dentro de los espacios de trabajo unas relaciones sociales que ponen en evidencia los intereses dispares del capital y del trabajo que dan cuenta, por un lado, del carácter conflictivo de estas relaciones y por otro lado, del ejercicio del poder, de la eficacia de las estrategias empresariales de dominación y de las acciones y fuerzas de resistencia que articulan y determinan las relaciones sociales en los espacios laborales.

Ante la pregunta: ¿Por qué estudiamos el poder, sus relaciones y efectos en el campo aplicado del conocimiento denominado psicología de las organizaciones?, Foucault ${ }^{8}$ plantea, en términos generales que la pregunta implica para las ciencias humanas en general su estatuto de cientificidad y sus prácticas sociales. En particular para los psicólogos una cuestión fundamental: la relación del sujeto y el poder como efecto de la verdad liberada, enunciada en un contexto sociocultural específico.

\section{Aproximación a la noción de poder en la teoría clásica}

Al revisar la literatura científica sobre la teoría clásica de la administración de las organizaciones, no es fácil establecer un parámetro universal de lo que es el poder en las

7. Andrés Rodríguez, «Poder y políticas en las organizaciones», Psicología Política, n. ${ }^{\circ} 5$ (1992): 99-115.

8. Michel Foucault, «El sujeto y el poder», Revista Mexicana de Sociología 50, n. 3 (1988): 3-20. 
relaciones humanas, sobre todo si se reconoce que son muchas las definiciones que se han dado a lo largo de la historia de las ciencias sociales y humanas, con intereses políticos e ideológicos, que pretenden legitimar ciertas modalidades de dominación y centradas en una visión puramente jurídica. En síntesis, si bien es cierto que el concepto de poder es complejo y difícil de precisar, entre los científicos sociales existe cierto consenso en torno a las dimensiones que constituyen el poder: la distinción entre poder y autoridad, el poder socializado frente al poder personalizado y las cinco fuentes clásicas del poder ${ }^{9}$.

Una definición ampliamente utilizada de poder, en el contexto organizacional, es la propuesta por Robert Dahl ${ }^{10}$, según este autor, una persona (A) tiene poder sobre otra persona, grupo o institución (B), en la medida en que puede conseguir que (B) haga o deje de hacer algo, que no lo haría de otro modo. Esta forma de entender el poder se estudió ampliamente en las décadas pasadas, asumiendo que el poder era algo exclusivo de la persona que lo ostentaba y de su posición en la estructura jerárquica de la organización social o empresarial. La aproximación ha sido abandonada en los últimos años por considerarla reduccionista y psicologicista, al no tener en cuenta aspectos del contexto sociocultural, económico y político en el que se enmarcan todas las relaciones laborales.

En el mismo sentido, para el sociólogo alemán Max Weber el poder, en términos sociológicos amplios hace referencia a una relación social caracterizada por la eventual imposición de la voluntad de uno sobre otros. Para este autor, el poder implica no solo un acto deliberado y consciente de imponer la voluntad de una de las partes sino fundamentalmente la imposición de una clase de intereses particulares y una relación de dominación y sumisión de unos sobre otros sin resistencia ni lucha, donde unos mandan y otros simplemente obedecen. Por esta razón define al poder como: «la probabilidad de imponer la propia voluntad, dentro de una relación social, aun contra toda resistencia y cualquiera que sea el fundamento de esa probabilidad $»^{11}$.

Desde una perspectiva más reciente y sociocultural, el poder se puede entender como el potencial de influencia que las personas ejercen o pueden ejercer en las organizaciones, sin importar su posición en la estructura jerárquica de una organización. En palabras del psicólogo social Edgar Schein, el poder hace referencia a la capacidad de influir sobre alguna persona o grupo con el fin de que acepten nuestras ideas» ${ }^{12}$.

Pfeffer define el poder como: «la habilidad potencial para influir sobre las conductas, cambiar el curso de las acciones, vencer las resistencias y hacer que las personas hagan cosas que las personas no harían de otro modo» ${ }^{13}$. Se enfatiza que el poder es una habilidad que poseen o adquieren las personas para influir o modificar la conducta de otros, pero no necesariamente sobre sus propias conductas. Para el autor, el poder no

9. Rodríguez, «Poder y políticas...», 99-115.

10. Robert Dahl (1957), citado por Lourdes Munduate, José Guerra e Inés Martínez, «El poder en los procesos de cambio», en: Carlos Guillen (coord.). Psicología del trabajo para las relaciones laborales (Madrid: McGraw Hill, 2001).

11. Max Weber, Economía y sociedad (México: Fondo de Cultura Económica, 1979), 43.

12. Edgar Schein, Psicología de la organización (México Prentice Hall Internacional, 1991), 29.

13. Pfeffer, Managing with power..., 30 . 
se relaciona con el autocontrol o la autorregulación del comportamiento emocional del directivo o administrador de los recursos humanos de una empresa.

Sintetizando los aspectos implícitos de las conceptualizaciones sobre el poder, señalan cuatro aspectos fundamentales: el poder es relacional, el poder surge de la dependencia de recursos, el poder implica libertad de conducta para tomar decisiones, y el poder es considerado como una capacidad potencial. Es decir, el poder puede existir sin que se utilice. En el mismo sentido, Munduate, Guerra y Martínez, plantean que el poder supone, al menos: a) la habilidad para dirigir o controlar los recursos humanos, informativos y materiales, a fin de conseguir una meta o unos resultados determinados; b) la habilidad de personas o grupos para conseguir resultados valorados por un sistema en el que otras personas o grupos también los desean para sí mismos; c) la capacidad de dominación implícita o explícita, y la capacidad de resistir al poder o la influencia de otros ${ }^{14}$.

Por su parte, Ragings y Sundstrom ${ }^{15}$ han identificado tres maneras posibles de comprender y definir el poder:

1. El poder entendido como una propiedad individual. Desde esta perspectiva, el poder es definido como la habilidad individual (percibida o no) de influir en las conductas de otras personas. Se asume el poder como un rasgo de personalidad o una habilidad adquirida que incrementa la fuerza potencial de influencia que proviene del interior del individuo.

2. El poder entendido como una propiedad de las relaciones interpersonales. La perspectiva asume que un individuo solo tiene poder cuando entra en relación con otro y en situaciones específicas. El análisis de las relaciones de poder se centra en los procesos diádicos y en las percepciones más que en las cualidades personales.

3. El poder entendido como una propiedad de la organización. La perspectiva define el poder como el acceso y control de una serie de recursos de la organización derivados de la división estructural del trabajo que dotan al individuo de la capacidad de ejercer influencia sobre los comportamientos de los otros.

Por otra parte, la inquietud sobre el poder en las organizaciones ha sido abordada en el trabajo de French y Raven ${ }^{16}$ quienes inicialmente realizaron una clasificación de cinco categorías sobre las bases o fuentes del poder social. La tipología toma en cuenta básicamente la influencia ejercida por un agente social (A), sobre una persona, grupo o institución (B). Las clases de poder según estos autores son: a) poder de recompensa, b) poder de coacción, c) poder legítimo, d) poder referente y e) poder experto.

Recientemente, Raven ${ }^{17}$, después de varios estudios sobre las dinámicas al interior de los grupos de trabajo, incorporó una sexta categoría como fuente de poder en los grupos

14. Lourdes Munduate, José Guerra e Inés Martínez, «El poder en los procesos de cambio», en Carlos Guillén (coord.). Psicología del trabajo para las relaciones laborales (Madrid: McGraw Hill, 2001).

15. Belle Ragins y Eric Sundstrom, «Gender and power in organizations: A longitudinal perspective», Psychological Bulletin 1 n. ${ }^{\circ} 105$ (1989): 51-88.

16. John French y Bertram Raven, Social Power (USA: University of Michigan Press, 1959).

17. Bertram Raven, «Influencia interpersonal: un modelo interactivo del poder y algunas aplicaciones», en Psicología política y procesos jurídicos (Barcelona: PPU, 1990). 
sociales, que denominó: poder informacional. Actualmente, se sigue estudiando cómo el poder de la información afecta positiva o negativamente las relaciones interpersonales y la efectividad del liderazgo gerencial.

Sin embargo, estudios recientes han establecido una distinción entre poder socializados (dirigido a ayudar a los demás) y poder personalizado (dirigido a ayudarse a sí mismo). Esta distinción, de cierto modo explica la razón por la que el poder tiene para muchas personas una connotación negativa, al considerar que los directivos y muchas otras personas en las organizaciones de trabajo asalariado, se preocupan principalmente por adquirir el poder personalizado para lograr sus propios fines y no para ayudar a los demás.

De acuerdo con Pelegrí ${ }^{18}$, las formas de poder más generalmente admitidas son: la coerción (ya sea basada en la fuerza, en la amenaza de sanciones o en la promesa de recompensas), la manipulación (si se consigue mediante engaño), el derecho (cuando se concede la autoridad) y la influencia (a veces calificada como persuasión). French y Raven (1959) incluyen unas modalidades que son relevantes para la aplicación en el trabajo social y los servicios sociales: el poder referente (el que produce identificación, deseo de ser como alguien que supone un modelo), poder de experto (cuando se tienen conocimientos teóricos o prácticos) y poder informacional (si se está en situación de controlar la información que afecta las decisiones).

Por su parte, Peiró y Meliá ${ }^{19}$ establecen dos categorías relacionadas con el poder de la posición ocupada dentro de una organización y el poder personal asociado al carisma y al poder referente, antes mencionado. En síntesis, para estos autores, el poder formal en las organizaciones está integrado por las bases del poder legítimo, coercitivo, de recompensa e informacional, asociados a la estructura social de la empresa y al rol desempeñado por el agente social. Mientras que el poder informal, agrupa las bases del poder experto y referente. No depende del rol desempeñado ni asignado en la estructura organizacional sino de características y atributos personales del agente social.

\section{El concepto de poder en la obra de Michel Foucault}

Más allá de la noción clásica del poder absoluto y soberano que restringe y se reduce a prohibir ciertas acciones, Michel Foucault nos ofrece un concepto más dinámico y productivo de regulación, control y disciplinamiento tanto del cuerpo como del alma humana en los contextos laborales de la sociedad industrial. Por lo tanto, actualmente el nombre de Michel Foucault, para muchos investigadores de las ciencias sociales, está estrechamente vinculado con los análisis del poder. Según Foucault, el análisis del poder se puede realizar desde dos paradigmas teóricos: el jurídico y el estratégico.

El paradigma jurídico ${ }^{20}$ concibe el poder a partir de la Ley y el Orden. La ley crea la regla de obediencia, y frente a las ineludibles transgresiones utiliza la represión mediante

18. Xavier Pelegrí Viaña, «El poder en el trabajo social: una aproximación desde Foucault», Cuadernos de Trabajo Social, n. ${ }^{\circ}$ 17, (2004): 21-43.

19. José Peiró y José Meliá, Formal and informal power in organizations: A bifactorial theory of power (EAWOP, 1999).

20. Michel Foucault, Un diálogo sobre el poder y otras conversaciones (Madrid: Alianza, 2002). 
el uso de la fuerza sobre el cuerpo o la negación de recompensas sociales. En este paradigma, de acuerdo con la propuesta de Ibáñez ${ }^{21}$, el poder se concibe como una posesión, que va en una sola dirección: de arriba hacia abajo; las instancias intermedias (escuela, familia, la empresa) reproducen el poder delegado; el poder controla y utiliza el saber, y se manifiesta en procedimientos de exclusión y de encierro.

Según este paradigma, el poder del maestro o del jefe en el ámbito laboral, se agota en el ámbito de la escuela o la fábrica-oficina, en la imposición del orden y la disciplina al alumno-obrero y en la correspondiente sanción. Fuera de la escuela, fábrica u oficina, el maestro o el jefe carecen de poder: pues no es su «jurisdicción». Sin embargo, el paradigma del poder ha venido funcionando tradicionalmente y ejerce un efecto de enmascaramiento en los individuos al debilitar su resistencia al poder.

Por otra parte, el paradigma estratégico es la propuesta de Foucault y se caracteriza, entre otras cosas, por las siguientes proposiciones:

1. El poder no es algo que se adquiera, se ejerce en el juego de relaciones móviles y no igualitarias; no existen zonas sin poder o que escapen a su control. Se puede decir que toda la sociedad es un complejo de relaciones de poder, o como dice Foucault: «el poder está en todas partes; no es que lo englobe todo, sino que viene de todas partes. El poder no es una institución, y no es una estructura, no es cierta potencia de la que algunos estarían dotados: es el nombre que se presta a una situación estratégica compleja en una sociedad dada ${ }^{22}$.

2. Para Michel Foucault, las relaciones de poder son inmanentes a otros tipos de relaciones (económicas, de conocimiento, sexuales), constituyen las condiciones y los efectos de desigualdades y desequilibrios en todos los intersticios del tejido social: «entre un hombre y una mujer, en una familia, entre un maestro y su alumno, entre el que sabe y el que no sabe, pasan relaciones de poder que no son la proyección pura y simple del gran poder del soberano sobre los individuos; son más bien el suelo movedizo y concreto sobre el que ese poder se incardina $»^{23}$.

3. El poder, a diferencia del paradigma jurídico, viene de abajo, no hay una matriz general que dé cuenta totalmente de las relaciones de poder; más bien se forman líneas de fuerza difusas por todo el sistema social que deben ser estudiadas, ya que adopta la forma de técnicas y promociona instrumentos de intervención material.

4. El poder no es total, ni tiene por qué ser siempre negativo (centrado en la represión), sino que puede manifestar un aspecto productivo porque promueve subjetividades particulares, regula y configura campos de posibilidad a través de las acciones y el saber: «lo que hace que el poder agarre, que se le acepte, es simplemente que no pesa solamente como una fuerza que dice no, sino que de hecho la atraviesa, produce cosas, induce placer, forma saber, produce discursos» ${ }^{24}$.

21. Tomas Ibáñez, Poder y libertad (Barcelona: Hora, 1983).

22. Foucault, Un diálogo sobre el poder..., 13.

23. Ibíd., 15.

24. Ibíd., 17. 
5. Donde hay relaciones de poder hay resistencias al poder, un contrapoder que se forma en su misma relación; que no se pueda estar fuera del poder no quiere decir que se está atrapado, la resistencia está en todas partes dentro de la red de poder: «existe porque está allí donde el poder está: es pues como él, múltiple e integrable en estrategias globales» ${ }^{25}$. Además, esta concepción también descubre la sutil transformación de las formas de represión hacia los signos que configuran la norma disciplinaria que moldea la mente; la disciplina es una fórmula general de dominación que se basa en el control minucioso del sujeto mediante el examen sistemático, la distribución espacial y la normalización.

\section{Relaciones de poder y psicología organizacional}

En términos generales se puede afirmar que a Foucault no le interesa el estudio del poder como un objeto o substancia, por el contrario, está muy interesado en las relaciones que el sujeto estable con el poder, incluso como dichas relaciones de poder producen un tipo de sujeto particular. En este sentido, una de las preguntas que más inquieta a Foucault, no es tanto qué es el poder, sino fundamentalmente cómo es ejercido el poder, ya que dicha inquietud unifica tres preguntas: ¿Qué es el poder? ¿Por qué medios es ejercido el poder? ¿Cómo se manifiesta? No se limita a describir los efectos del poder sin siquiera relacionar estos efectos tanto a sus causas como a su naturaleza básica. En este sentido, le interesa analizar qué sucede cuando los individuos ejercen (como ellos dicen) el poder sobre otros.

Respecto a esta forma de entender el poder, es necesario distinguir, de acuerdo con Foucault, en primer lugar el que se ejerce sobre las cosas y proporciona la capacidad de modificarlas, utilizarlas, consumirlas o destruirlas. Es decir, un poder que surge de aptitudes directamente inscritas en el cuerpo o que se transmite mediante instrumentos externos. Por otra parte, lo que caracteriza el poder es que expresa relaciones entre individuos (o entre grupos). En consecuencia, si hablamos de estructuras o de mecanismos de poder, es sólo en la medida en que suponemos que ciertas personas ejercen poder sobre otras.

En este contexto, para Foucault, es necesario distinguir las relaciones de poder de las relaciones de comunicación que transmiten una información por medio de un lenguaje, un sistema de signos o cualquier otro medio simbólico. La comunicación es siempre, sin duda, una cierta manera de actuar sobre el otro o los otros, pero la producción y la circulación de elementos del significado pueden tener como objetivo o como consecuencia ciertos efectos de poder En consecuencia, las relaciones de poder poseen una naturaleza específica, pasen o no a través de sistemas de comunicación. No deben confundirse entonces las relaciones de poder, las relaciones de comunicación y las capacidades objetivas.

De acuerdo con la filósofa Esther Díaz ${ }^{26}$, las relaciones de poder implican acciones sobre acciones: incitar, inducir, desviar, facilitar, dificultar, ampliar o limitar. Las relaciones de poder se caracterizan por la capacidad de «unos» para poder «conducir» las

25. Ibíd., 18

26. Esther Díaz, La filosofía de Michel Foucault (Buenos Aires: Biblos, 2003). 
acciones de otros. En consecuencia, las relaciones de poder son un tipo de relación estratégica entre acciones, es decir, entre sujetos de acción, que modifica las fuerzas en relación.

En síntesis, las relaciones de poder constituyen sistemas de fuerzas regulados y concertados, que a través de la disposición de sus espacios y de los reglamentos regulan la vida interna de las personas en las distintas actividades en las que se organizan en un contexto social marcado por la división del trabajo y la jerarquía de tareas.

El ejercicio del poder es un modo de relacionarse en el que ciertas acciones de unas personas modifican las acciones o conductas de otras, lo cual significa que no existe algo llamado el poder, con o sin mayúsculas, que exista universalmente, en forma masiva o difusa, concentrado o distribuido. Solo existe el poder que ejercen «unos» sobre «otros».

Foucault ${ }^{27}$ plantea que el poder solo existe en acto. Es lo que hacemos cuando interactuamos, aunque, desde luego, se inscriben en un campo de posibilidades dispersas. Lo cual significa que el poder no es una especie de consentimiento. En sí mismo, el poder no es renunciar a la libertad ni mucho menos la cesión de derechos. La relación de poder puede ser el efecto de un consentimiento permanente o anterior, pero no es por naturaleza la manifestación de un consenso.

En síntesis, para el autor, una relación de poder se articula sobre dos elementos, ambos indispensables para ser justamente una relación de poder: que «el otro» (aquel sobre el cual éste se ejerce) sea totalmente reconocido y que se le mantenga hasta el final como un sujeto de acción; y que se abra, frente a la relación de poder todo un campo de respuestas, reacciones, efectos y posibles invenciones. Por tanto, el ejercicio del poder es un conjunto de acciones sobre acciones posibles; opera sobre el campo de posibilidad o se inscribe en el comportamiento de los sujetos actuantes: incita, induce, seduce, facilita o dificulta; amplía o limita, se vuelve más o menos probable; de manera extrema, constriñe o prohíbe de modo absoluto; con todo, siempre es una manera de actuar sobre un sujeto actuante o sobre sujetos actuantes, en tanto que actúan o son susceptibles de actuar.

En última instancia, como señala Foucault, cuando se define el ejercicio del poder como un modo de acción sobre las acciones de los otros, cuando se caracterizan estas acciones por el «gobierno» de los hombres, de los unos por los otros -en el sentido más amplio del término- se incluye un elemento importante: la libertad. El poder se ejerce únicamente sobre «sujetos libres» y sólo en la medida en que son «libres». Por esto queremos decir sujetos individuales o colectivos, enfrentados con un campo de posibilidades, donde pueden tener lugar diversas conductas, diversas reacciones y diversos comportamientos.

\section{Psicología organizacional}

Es importante resaltar que la constitución del campo de conocimiento que actualmente se denomina psicología de las organizaciones, es realmente reciente, oficialmente se puede señalar que en 1970 la American Psycological Association (APA) incluye este campo en su división 14. Sin embargo, no debemos olvidar que su antecedente más cercano es la psicología industrial. En este orden de ideas, es oportuno señalar que «la

27. Foucault, Un diálogo sobre el poder... 
psicología industrial/organizacional es un invento del siglo $\mathrm{XX}{ }^{28}$, además se afirma que los psicólogos experimentales fueron los más interesados en aplicar los nuevos principios de la psicología a los problemas de las organizaciones. En consecuencia, los primeros trabajos en las organizaciones industriales de esa época, se enfocaron en asuntos relacionados con el desempeño laboral y la eficiencia organizacional ${ }^{29}$.

El interés de los psicólogos norteamericanos de comienzos del siglo XX por estudiar ciertos aspectos y temas de la vida laboral, estuvo muy influenciado por el pensamiento del ingeniero industrial Frederick Taylor (1911) expuesto en su libro sobre la administración científica. El texto incluye varios principios para guiar las prácticas administrativas de las organizaciones industriales, fundamentalmente hacia el control de la productividad de los empleados en las fábricas.

La influencia de las ideas de Taylor en las prácticas y ejercicio de la psicóloga industrial, sigue siendo valiosa en nuestros días y se puede apreciar fácilmente en cuatro de los principios de la administración científica, de acuerdo con Spector ${ }^{30}$ :

1. Cada puesto ha de analizarse con mucho cuidado para poder especificar la manera óptima de realizar cada tarea.

2. Se debe seleccionar (contratar) a los empleados de acuerdo con las características relacionadas con el desempeño del puesto. Los gerentes necesitan estudiar a los empleados existentes, para encontrar qué características personales son importantes.

3. Es preciso capacitar con cuidado a los empleados, para que realicen las tareas de su puesto.

4. Es necesario recompensar a los empleados por su productividad, para alentar niveles de desempeño más altos.

Como se puede apreciar, la mayor parte de los esfuerzos de los psicólogos industriales del siglo XX, se dirigen al fortalecimiento de la efectividad y el funcionamiento de las organizaciones. Po tanto, su labor se enfoca principalmente en la selección de las personas que puedan realizar mejor el trabajo, en la capacitación para un buen desempeño, en la construcción de puestos de trabajo que faciliten las tareas o los diseños de organizaciones que funcionen de manera óptima. En consecuencia, la psicología del trabajo industrial/organizacional llega desde sus inicios en Norteamérica, con sus discursos y prácticas de selección de los trabajadores más aptos para responder a una demanda del mercado laboral.

Por el momento podemos decir que en el campo de la psicología industrial hay dos aspectos de suma importancia: el primero abarca el estudio del lado humano de las

28. Paul Spector, Psicología industrial y organizacional: investigación y práctica (México: Manual Moderno, 2002), 7.

29. Néstor Porras Velásquez, «Aproximación histórica a la psicología del trabajo y de las organizaciones en Colombia». Revista Interamericana de Psicología /Interamerican Journal of Psychology (IJP) 50, n. ${ }^{\circ} 3$ (2016): 317-330.

30. Spector, Psicología industrial y organizacional...,7 
organizaciones. Muchos psicólogos, en especial aquellos que son profesores en universidades, dirigen investigaciones sobre las personas en ambientes laborales. El segundo, incluye la aplicación de los hallazgos de dichos estudios, en la solución de los problemas humanos que surgen en las organizaciones. Por esta razón, muchos psicólogos están vinculados a las organizaciones industriales o empresariales, en calidad de empleados o como consultores.

Asimismo, el psicólogo Paul Spector sostiene que: «la psicología industrial/organizacional es un pequeño campo de la psicología aplicada que se refiere al desarrollo y aplicación de los principios científicos en el lugar de trabajo» ${ }^{31}$. Además, señala que: uno de los principales objetivos de la psicología industrial/organizacional es ayudarles a funcionar de manera más efectiva; para lograrlo se debe contar con descubrimientos científicos en los cuales fundamentar su práctica, Sin embargo, el autor reconoce que no toda investigación en el campo se hace con la intención de llevarla a la práctica, ya que algunos psicólogos-investigadores estudian el comportamiento laboral con el único propósito de saber por qué las personas presentan un comportamiento específico en su trabajo. Es decir, las preguntas de investigación se circunscriben a dar respuestas inmediatas a situaciones o problemas muy concretos de la realidad organizacional ${ }^{32}$ de cada empresa en particular. Este proceder configura a mediano y largo plazo una forma singular de pensar y actuar denominada cultura organizacional ${ }^{33}$.

Todos estos cambios han tenido un impacto importante en el rol desempeñado y asignado a los psicólogos en el ámbito de las organizaciones, igualmente se han modificado sus funciones y responsabilidades en el mundo del trabajo, así como los modelos conceptuales y las técnicas que utiliza en su actividad profesional cotidiana. Sin embargo, la psicología como disciplina madre, con sus métodos y teorías científicas, sigue siendo la base conceptual de la psicología organizacional. Por esta razón, el psicólogo ha tenido que ampliar su actividad a aspectos relacionados con otras disciplinas como la economía, el derecho laboral, la medicina del trabajo, entre otras, tanto cuando trabaja de forma individual como en equipos multidisciplinares en la solución de problemas humanos en las organizaciones laborales ${ }^{34}$.

Tradicionalmente se ha asumido una perspectiva puramente personal-individual que pretende analizar cómo la gente común piensa, siente y valora su papel en el sistema de empleo y qué impacto psicosocial produce en ella su situación laboral (de empleo, subempleo o desempleo). Desde una perspectiva más sociocultural la mirada del psicólogo se focaliza en los aspectos psicosociales específicos de la actividad laboral en un contexto organizacional.

31. Paul Spector, Psicología industrial..., 4.

32. Néstor Porras Velásquez, «La realidad organizacional desde la perspectiva psicosocial», Revista Iberoamericana de Psicología: Ciencia y Tecnología 5, n. ${ }^{\circ} 1$ (2012): 7-18.

33. Néstor Porras Velásquez, «Elementos básicos para el análisis de la cultura de las organizaciones desde la psicología», Tesis psicológica 4, n. $^{\circ} 1$ (2009): 36-56.

34. Néstor Porras Velásquez «¿Qué es lo que hace exactamente un psicólogo organizacional? Una reflexión crítica desde las teorías del rol», Poiésis, n. ${ }^{\circ} 25$ (2013). 
Por el momento, basta señalar la multiplicidad de aspectos abordados por la psicología del trabajo, así como la diversidad de enfoques utilizados para el análisis de la conducta de las personas en el trabajo. Sin embargo, se aprecia una ausencia de interés por estudiar el tema de las relaciones de poder al interior de las organizaciones empresariales por parte de los psicólogos especializados en el área. Quizá, por esta razón muchos psicólogos autodenominados «prácticos» asumen su labor profesional como verdaderos «administradores» de recurso humanos y no como psicólogos del trabajo y de las organizaciones, buscando su identidad y reconocimiento profesional en otros campos disciplinares. El comportamiento obedece tal vez a evitar, consciente o inconscientemente, el problema ético del ejercicio del poder sobre sí mismos o sobre los otros, y principalmente a no pensar en las relaciones de dominación y sumisión que el ejercicio del poder genera en los contextos laborales.

\section{El poder disciplinario dispositivo de control administrativo}

El individuo es sin duda el átomo ficticio de una representación ideológica de la sociedad; pero es también una realidad fabricada por esta tecnología específica de poder que se llama la disciplina.

M. Foucault

En términos amplios, el concepto de dispositivo ha sido abordado por diversas disciplinas, básicamente provenientes de las ciencias sociales y humanas. Algunos autores, entre estos Foucault, coinciden en señalar que el dispositivo funciona en el contexto de las relaciones de poder y la construcción de relaciones entre los sujetos. Para Foucault, el teórico mas reconocido, el dispositivo es la red que puede establecerse entre un conjunto heterogéneo de elementos (incluye discursos, instituciones, reglamentos, leyes, medidas administrativas, enunciados científicos, proposiciones filosóficas y morales), con una función estratégica concreta e inscrito en una relación de poder.

Giorgio Agamben, ${ }^{35}$ llama dispositivo a todo aquello que tiene, de una manera $u$ otra, la capacidad de capturar, orientar, determinar, interceptar, modelar, controlar y asegurar los gestos, las conductas, las opiniones y los discursos de los seres vivos. Por su parte, García Fanlo, ${ }^{36}$ asume que el dispositivo es un régimen social productor de subjetividad; es decir, productor de sujetos-sujetados a un orden del discurso, cuya estructura sostiene un régimen de verdad.

Yela e Hidalgo, ${ }^{37}$ sostienen que la disciplina y la vigilancia son los dispositivos que han hecho posible la existencia y perpetuidad de las relaciones de poder en el tejido

35. Giorgio Agamben, «¿Qué es un dispositivo?», 2006, http://ayp.unia.es/r08/IMG/pdf/agamben-dispositivo. pdf

36. Luis García Fanlo, «¿Qué es un dispositivo? Foucault, Deleuze, Agambem», A Parte Rei. Revista de Filosofía, n. ${ }^{\circ} 74$ (2011), http://ayp.unia.es/r08/IMG/pdf/agamben-dispositivo.pdf 
mismo de la sociedad, ya que garantizan tanto la obediencia como la economía de los gestos y el tiempo, y también la presencia del poder en el núcleo de las relaciones entre los individuos. En consecuencia, se puede llamar disciplinas a: «los métodos que permiten el control minucioso de las operaciones del cuerpo que garantizan la sujeción constante de sus fuerzas y les imponen una relación de docilidad-utilidad ${ }^{38}$.

La existencia de la disciplina permite fabricar cuerpos sometidos y ejercitados, es decir, cuerpos dóciles; que incrementan las fuerzas, en términos económicos de utilidad y las disminuyen, en términos políticos de obediencia. En síntesis, la disciplina es: «una anatomía política el detalle ${ }^{39}$. Por tanto, el poder disciplinar obtiene sus mayores logros empleando instrumentos simples como: la vigilancia jerárquica, la sanción normalizadora y el examen. Por esta razón, Foucault se refiere a la vigilancia jerárquica, continua y funcional que hace del poder disciplinario un poder múltiple, anónimo y automático, que funciona en red.

Por otra parte, ¿en qué consisten las tecnologías del yo? En términos generales, son las practicas que los individuos aplican sobre sí mismos. Resultan de la reflexión sobre los modos de vida, sobre las relaciones de existencia, sobre los modos de regular la propia conducta, de fijarse medios y fines ${ }^{40}$. En otras palabras, las tecnologías del yo son «procedimientos, existentes sin duda en cualquier civilización, que son propuestos o prescriptos a los individuos para fijar su identidad, mantenerla o transformarla en función de un cierto número de fines, y todo ello gracias a las relaciones de dominio de sí sobre uno mismo o de conocimiento de uno por sí mismo» ${ }^{41}$. Queda claro que las tecnologías del yo nos permiten ver cómo los hombres se gobiernan a sí mismos en los espacios propios del mundo del trabajo.

Ante la pregunta: ¿Qué acciones debe llevar a cabo el individuo sobre su emocionalidad para hacer que su comportamiento concuerde con las reglas o normas del trabajo?, la respuesta, según Marcela Zangaro, debe estar orientada a satisfacer el doble carácter del trabajo ético: ser constructor y reparador de sus emociones y estados afectivos. Por esta razón, en este contexto se amalgaman la identidad entre vida y trabajo, que borra la línea que separa el trabajo del no trabajo. De acuerdo con Zangaro ${ }^{42}$, el trabajo ya no es un medio para un fin, pues desaparece la relación instrumental. El trabajo es el fin mismo porque es la vida misma. En consecuencia, se puede afirmar que en el actual contexto sociocultural y económico, el individuo no se define por su actividad: sino que el individuo es su actividad y su actividad es su empleo.

Finalmente, al asumir las organizaciones como sistemas funcionales, suponemos que estos sistemas se unen mediante relaciones de un poder que se ejerce y se acepta. Significa

37. Jeisson Yela y Clara Hidalgo, «El poder en Foucault: bases analíticas para el estudio de las organizaciones», Cuadernos de Administración. Universidad del Valle, $n^{\circ} 44$ (2010): 57-69.

38. Foucault, Un diálogo sobre el poder..., 141.

39. Michel Foucault, Estrategias de poder (Vol. II. Madrid: Alianza, 2001), 143.

40. Foucault, Un diálogo sobre el poder...

41. Foucault, Un diálogo sobre el poder..., 55.

42. Marcela Zangaro, «Subjetividad y trabajo: el management como dispositivo de gobierno». Trabajo y Sociedad 15, n. $^{\circ} 16$ (2011): 163-177. 
que, el poder como estrategia en cualquier relación social-laboral, se debe considerar ante todo como acción de unos agentes hacia otros. No obstante, las relaciones de poder en el campo de las organizaciones adquieren un nuevo escenario cuando se habla de Gestión del Conocimiento, ya que el conocimiento, convertido en un saber que todos los miembros de la organización deben aceptar, y que cumple con los criterios de verdad producidos e impuestos por la fuerza del poder dominante en un momento determinado, pueden cambiar en el juego dialéctico y dinámico de resistir e imponer la verdad producida como realidad organizacional.

\section{Conclusiones}

Las prácticas y relaciones de poder en el mundo del trabajo no han tenido la acogida ni el interés sobre otros temas en el campo de la psicología organizacional, por parte de los psicólogos y psicólogas que investigan, practican o aplican el saber psicológico en los ámbitos organizacionales y empresariales de la era industrial. La falta de interés refleja un silencio cómplice, una supuesta neutralidad valorativa, que a partir de un saber y un saber hacer psicológico, parece más orientado por un conjunto de ideas preconcebidas acerca de la relación entre la autoridad, el autoritarismo y la obediencia, además del conformismo profesional con el rol asignado.

En general, el poder social es asumido como la capacidad de controlar, modificar o influir en el comportamiento de otra persona. Por esta razón, no se puede decir solamente que el poder reprime, prohíbe y limita el comportamiento de las personas y los grupos humanos, sino que el poder controla y regula, vigila y gestiona. Ya no encierra ni excluye como en épocas anteriores sino que cura; es decir, vuelve «normal» a las personas y grupos. Por tanto, el poder no puede ser entendido solamente como un acto social contingente que depende de imponer la propia voluntad, sino que el poder constituye un tipo de vínculo propio del psiquismo humano como lo han venido proponiendo varios autores.

En el mundo socio laboral, mediante el poder se puede lograr que otra persona haga lo que yo quiero. Así, no hay duda que el poder es, ante todo, una instancia productiva. El poder produce saber, engendra procedimientos y objetos de saber. Quien ocupa una posición de poder produce saber. En el contexto, la interrogante que surge es por el saber producido por la psicología y sus prácticas de gestión humana. Como señala Foucault (1988), el poder logra su efecto y es aceptado porque produce verdades para las personas, proporciona subjetividades que la gente puede asumir en su vida cotidiana, configura identidades y regula las visiones del mundo. Así es cómo el saber y el poder se constituyen. En pocas palabras, «el ejercicio del poder requiere de la verdad y de los diferentes mecanismos para producirla $\rangle^{43}$.

En definitiva, las relaciones de poder no solo dictan en proceder en el trabajo y en la vida sino también cómo se debe ser. En consecuencia, con el dispositivo psicológico, en términos de un discurso que promueve ciertas prácticas de gestión humana, estamos sometidos a la producción de la verdad desde el poder y no podemos ejercerlo más que a través de la producción de la verdad. De tal forma que el poder necesita «producir la

43. Foucault, Un diálogo sobre el poder..., 103. 
verdad» para funcionar. De ahí que la verdad psicológica en el mundo del trabajo se haga ley, construya el discurso de lo verdadero en cuanto a las prácticas de gestión humana, al menos en parte, cuando promueve efectos de poder y sujeción de las voluntades y las conciencias de los miembros de las organizaciones productivas.

\title{
ABSTRACT
}

\section{Relationships of Power and Psychology of Organizations: a reflection from Foucault}

\begin{abstract}
This article's objective is to critically examine the contribution of Foucault's proposal for the analysis of power relationships in the psychology of organizations. The methodology used was a documental analysis with conceptualization of power and the bases for their foundation; the ways they are executed and some applications and implications of these models in the management of human resources. Results show practices of human management as control mechanisms and coordinating of the subjectivist methods of the individuals and the actions these individuals practice over themselves, using technologies of the self and the practices of administration over body and mind. Conclusions evidence the fundamental role of the manufacturing disciplinary mechanism in the construction and constitution of well-trained labor subjectivities.
\end{abstract}

Key words: organizational psychology, power relationships, technology of the self, control mechanisms, labor subjectivities.

\section{RÉSUMÉ}

\section{Les relations de pouvoir et la psychologie dans les organisations: une réflexion d'après Foucault}

Cet article a pour but de faire une révision critique des apports de la proposition de Foucault afin d'analyser les relations de pouvoir dans le champ de la psychologie des organisations. La méthode employée a été l'analyse documentaire des œuvres qui abordent les concepts de de pouvoir et les bases que les soutiennent, les manières de l'exercer et certaines applications et implications des modèles de la gestion des ressources humaines. Les résultats mettent en évidence ce dispositif de contrôle et l'articulateur des modes de subjectivation des individus, et les actions qu'ils exercent sur eux-mêmes, à travers des technologies du «je» et les pratiques de contrôle sur le corps et l'esprit. Les conclusions exposent le rôle fondamental du dispositif disciplinaire manufacturier dans la construction et constitution des subjectivités du travail «dociles».

Mots clés: Psychologie organisationnelle, relations de pouvoir, technologies du «Je», dispositifs disciplinaires de contrôle, subjectivités du travail.

\section{Bibliografía}

Agamben, Giorgio. «¿Qué es un dispositivo?». Roma: Edizioni Ottetempo. Versión en castellano, 2006. http:// ayp.unia.es/r08/IMG/pdf/agamben-dispositivo.pdf

Díaz, Esther. La filosofía de Michel Foucault. Buenos Aires: Biblos, 2003.

Foucault, Michel. El sujeto y el poder. Revista Mexicana de Sociología 50, n. ${ }^{\circ} 3$ (1988): 3-20.

Vigilar y castigar: nacimiento de la prisión. Madrid: Siglo XXI, 2005.

Estrategias de poder. Vol. II. Madrid: Alianza, 2001. 
Un diálogo sobre el poder y otras conversaciones. Madrid: Alianza, 2002.

French, John y Bertram Raven. Social Power. University of Michigan Press, 1959.

García, Luis. «¿Qué es un dispositivo? Foucault, Deleuze, Agambem». A Parte Rei. Revista de Filosofía n. ${ }^{\circ} 74$ (2011). http://serbal.pntic.mec.es/ cmunoz11/fanlo74.pdf

Rodríguez, Andrés y José González. «El poder y las políticas en las organizaciones». En. A. Rodríguez (Coord.) Introducción a la psicología del trabajo y de las organizaciones. (179-188). Madrid: Pirámide, 2008.

Ibáñez, Tomas. Poder y libertad. Barcelona: Hora, 1983.

Mintzberg, Henry. Power in and around organizations. Englewood Clifts, NJ: Prentice-Hall, 1993.

Munduate, Lourdes, José Guerra e Inés Martínez. «El poder en los procesos de cambio». En: Carlos Guillen (coord.). Psicología del trabajo para las relaciones laborales. Madrid: McGraw Hill, 2001.

Peiró, José y José Meliá. Formal and informal power in organizations: A bifactorial theory of power. EAWOP, 1999.

Pelegrí, Xavier. «El poder en el trabajo social: una aproximación desde Foucault». Cuadernos de Trabajo Social, n. ${ }^{\circ} 17$ (2004): 21-43.

Pfeffer, Jefrey. Managing with power. Boston: Harvard Business School Press, 1992.

Porras, Néstor. «Elementos básicos para el análisis de la cultura de las organizaciones desde la psicología». Tesis psicológica 4, n. 1 (2009): 36-56.

«La realidad organizacional desde la perspectiva psicosocial». Revista Iberoamericana de Psicología: Ciencia y Tecnología 5, n. ${ }^{\circ} 1$ (2012): 7-18.

«Inserción laboral y salud mental: una reflexión desde la psicología del trabajo». Tesis psicológica 8, n. ${ }^{\circ} 2$ (2013): 98-117.

«¿Qué es lo que hace exactamente un psicólogo organizacional? Una reflexión crítica desde las teorías del rol». Poiésis, n. ${ }^{\circ} 25$ (2013).

«La responsabilidad social empresarial e inserción laboral del enfermo mental». En Sonia Cogollo. Responsabilidad social Perspectivas para la acción en Colombia, 88-105, Medellín: FUNLAM, 2015.

«La Psicología del trabajo en relación con la subjetividad, la inserción laboral y la salud mental». En Salud mental y el sistema de producción, 61-101. Bogotá: Fundación universitaria los Libertadores, 2016.

«Aproximación histórica a la psicología del trabajo y de las organizaciones en Colombia». Revista Interamericana de PsicologialInteramerican Journal of Psychology (IJP) 50, n. ${ }^{\circ} 3$ (2016): 317-330.

Ragins, Belle y Eric Sundstrom. «Gender and power in organizations: A longitudinal perspective». Psychological Bulletin 1, n. ${ }^{\circ} 105$ (1989): 51-88.

Raven, Bertram. «Influencia interpersonal: un modelo interactivo del poder y algunas aplicaciones». En: I. Balaguer. Psicología política y procesos jurídicos. Barcelona: PPU, 1990.

Rodríguez, Andrés. «Poder y políticas en las organizaciones». Psicología Política, n. ${ }^{\circ} 5$ (1992): 99-115.

Schein, Edgar. Psicología de la organización. México: Prentice Hall Internacional, 1991.

Spector, Paul. Psicología industrial y organizacional: investigación y práctica. México: Manual Moderno, 2002.

Weber, Max. Economía y sociedad. México: Fondo de Cultura Económica, 1979.

Yela, Jeisson y Clara Hidalgo. «El poder en Foucault: bases analíticas para el estudio de las organizaciones». Cuadernos de Administración. Universidad del Valle, n. ${ }^{\circ} 44$ (2010): 57-69.

Zangaro, Marcela. «Subjetividad y trabajo: el management como dispositivo de gobierno». Trabajo y Sociedad, 15, n. ${ }^{\circ} 16$ (2011): 163-177. 